\title{
Incontinencia Pigmentaria. Consideraciones odontoestomatológicas: Profilaxis y terapéutica
}

\author{
Pipa Vallejo A*, González García M**, López-Arranz Monje E*, Fernández Toral J****
}

\begin{abstract}
RESUMEN
La incontinencia pigmentaria es una rara enfermedad, familiar, transmisión genética ligada al cromosoma X, de aparición casi exclusiva en mujeres, afectación cutánea muy típica y anomalías que afectan al pelo, dientes, ojos, sistema nervioso central, con una importante incidencia en las estructuras estomatognáticas tanto cuantitativa como cualitativamente. Los dientes se ven afectados en el $50-80 \%$ de los pacientes. Existe un retraso en la erupción dental, ausencia parcial o total de dientes, formas anormales de los mismos, dientes impactados, maloclusiones. Por tanto, se considera importante la prevención en estos pacientes insistiendo en la higiene oral, control de caries, revisiones orales periódicas, etc.. Se hace una exposición de las distintas opciones de tratamiento para obtener una armonía, una funcionalidad y estética dental aceptables.
\end{abstract}

Palabras claves: Incontinencia pigmentaria. Síndrome de Bloch-Sulzberger. Agenesias dentales. Prevención.

\section{SUMMARY}

Incontinentia pigmenti is a rare, family disorder of genetic transmisión linked to the X chromosome which appears almost exclusively in females; it causes skin disorders and anomalies that affect hair, teeth, eyes and the central nervous system. Teeth are affected in $50-80 \%$ of patients. Patients are late in teething, some or all teeth may be lacking, those that exist may have malformations, maloclusions. Therefore prevention is of ut most importance in these patients, with special attention given to dental hygiene, control of tooth decay, regular check-ups, etc..The different options for treatment will be examined in order to obtain acceptable dental harmony, functioning and aesthetics.

Key words: Incontinentia pigmenti. Bloch-Sulzberger syndrome. Dental agenesis. Prevention.

Aceptado para publicación: Noviembre 2004.

* Escuela de Odontología. Facultad de Medicina. Universidad de Oviedo.

** Servicio de Cirugía Oral y Maxilofacial del Hospital Universitario Central de Asturias.

*** Unidad de Genética del Hospital Universitario Central de Asturias.

Pipa Vallejo A, González García M, López-Arranz Monje E, Fernández Toral J. Incontinencia Pigmentaria. Consideraciones odontoestomatológicas: Profilaxis y terapéutica. Av. Odontoestomatol 2005; 21-4: 211-215.

\section{INTRODUCCIÓN}

Dentro del grupo de facomatosis con discromias extensas se encuentra la incontinencia pigmentaria(IP), enfermedad neurocutánea genética rara, que afecta a la piel, pelo, dientes, visión y SNC.
Descrita por Garrod, en 1906 como “...una variedad de idiocia mongólica con una pigmentación peculiar", la primera descripción completa es atribuida a Bardach, Bloch, Siemens y Sulzberger, durante la década de 1920. 
Franceschetti y Jadassohn, dividen la IP en: a) Síndrome de Bloch-Sulzberger y b) Displasia ectodermal tipo Naegeli .

La IP es hereditaria ligada al cromosoma X con carácter dominante, las mujeres tienen síntomas de gravedad variable, los varones no sobreviven después del parto, salvo casos con síndrome de Klinefelter. (1-3)

Se conocen dos loci en el cromosoma $\mathrm{X}$ asociados a IP:

\section{-IP1 en Xp11.21-cen.}

-IP2 (más del 95\%) en Xq28. En este locus se encuentra el gen NEMO ( NF-kB Esencial Modulador) a cuyas mutaciones se atribuye la enfermedad (4): codifica la proteina NEMO moduladora de la actividad del factor de transcripción NF-kB que interviene en el control del crecimiento del epitelio estratificado, permite a las células responder a estímulos externos, ...

La mutación más frecuente -80\% - consiste en una deleción de parte del gen. Más del $95 \%$ son pacientes del sexo femenino.

\section{OBSERVACIÓN CLÍNICA}

Niña de 8 años enviada desde su Centro de Salud al Servicio de Cirugía Maxilofacial para valoración, tratamiento y seguimiento de las anomalías maxilares, dentales, etc., que presenta.

Antecedentes familiares: Madre con enfermedad dermatológica no filiada y ausencia congénita parcial de dientes que precisó de tratamiento protésico. Resto sin interés

Antecedentes personales: Embarazo y parto sin incidencias dignas de mención. Presentó al nacer un cuadro dermatológico diseminado, predominio en miembros inferiores, múltiples lesiones vesículoampollosas, tratado con antibioterapia, que se suspendió al confirmar la negatividad bacteriológica. Desde su nacimiento, constatan áreas de alopecia difusa sin eritema ni descamación que remiten parcialmente; persistiendo áreas hipopigmentadas.

Analítica: los hemogramas realizados son normales, excepto una marcada eosinofilia.

Biopsia cutánea: incontinencia pigmentaria.

Revisión oftalmológica: dentro de la normalidad.

Exploración específica: Retraso en la erupción dentaria, tanto decidual como definitiva, constatando formas anormales en varios de ellos (dientes conoides, tamaño pequeño, cúspides accesorias). Paladar ojival.

Ortopantomografía: Ausencia de múltiples dientes, predominantemente en hemiarcada inferior izquierda.

Estudio de modelos: mordida cruzada anterior.

Cefalometría: Algunos parámetros de clase I, otros nos indican cierta tendencia a clase III esquelética.

\section{DISCUSIÓN}

Para el diagnóstico clínico son objetivables unos criterios mayores y menores (5-9) :

\section{-criterios mayores:}

$1^{\circ}$ estadio (vesículoampolloso). Fase vesicular, ampollosa, base eritematosa, distribución siguiendo las líneas de Blaschko (10), en extremidades, tronco, etc.

$2^{\circ}$ estadio (verrugoso). Caracterizado por lesiones pustulares, liquenoides, verrugosas, queratósicas, disqueratósicas.

$3^{\circ}$ estadio (pigmentado). Máculas hiperpigmentadas marrón oscuro, gris pizarra o arena tostada a lo largo de las líneas de Blaschko en tronco y extremidades, se conocen como "dermatosis en salpicadura".

$4^{\circ}$ estadio o de cicatrizado. Fase de la atrofia; cicatrices como manchas pálidas, máculas hipomelánicas, en pantorrillas, a veces la única presentación en el adulto. 


\section{-criterios menores:}

- pelo, pérdida de pelo en la corona de la cabeza. La alopecia es de tipo trófico, cicatricial (pseudopelada de Brocq). Agenesia de cejas y aplasia parcial de glándulas sudoríparas (11).

- uñas, en manos y pies, afectación en el $40 \%$ de los casos, forma transitoria, leve, presentan arrugas, hoyos, onicogriposis, están rotas.

- visión, el 90\% no presentan alteraciones oculares, la miopía o la hipermetropía son comunes; la coriorretinitis exudativa, es la patología clásica. También estrabismo, cataratas, atrofia óptica, desprendimiento de retina y fibrosis retrolental (12).

- sistema nervioso central, con retraso mental, psicomotor, edema cerebral, hidrocefalia, atrofía cerebral, tetraplejia espástica, epilepsia, actividad motora reducida, etc.

- dientes, se ven frecuentemente alterados, 50-80\%, de forma llamativa e importante para el diagnóstico, tanto la dentición temporal como la definitiva. Es habitual retraso en la erupción de los dientes (18\%), ausencia parcial de dientes (agenesia), formas anormales: conoidal, trapezoidal, en clavija, tamaño pequeño, cúspides accesorias. Radiograficamente es objetivable la impactación o la inclusión de algún diente (13). Las anomalías más comunes son la falta parcial de dientes y las formas anormales (14).

Hay tendencia a clase III, por rotación hacia arriba de la mandíbula, al intentar la acomodación oclusal por la ausencia de dientes y producirse la migración de dientes al existir numerosos espacios edéntulos. Esto produce disminución en la dimensión vertical y facilita la rotación mandibular $(15,16)$. La constitución y estructura interna del diente y esmalte es normal.

-Otras anomalías, estatura corta, hemivértebras, paladar ojival, espina bífida, luxación de cadera, sindactilia, condrodistrofia, fisura labial, anomalías cardíacas, genitourinarias, del desarrollo de las mamas (pezones extra, ausencia de pechos). La incidencia de estos síntomas es diez veces mayor que en la población general.
El diagnóstico se establece a partir de: criterios clínicos (sarpullido en recién nacida, eritema, vesículas, ampollas, hiperpigmentación ); sangre (elevación de $\mathrm{IgE}$, leucocitosis, eosinofilia, defecto en la quimiotaxis); estudios anatomopatológicos ( granos de melanina libres en la zona de hiperpigmentación, la " espongiosis eosinofílica", la disqueratosis ); análisis genético molecular ( el estudio molecular del gen IKBKG (NEMO) locus Xq28).

\section{Abordaje estomatológico}

Se debe extremar el cuidado de la salud bucodental por la ausencia de dientes: una exquisita higiene oral, esmerado cepillado dental, lingual, de forma pormenorizada, cepillos blandos, realizando movimientos suaves, sentido vertical, circular, procurar el uso de seda dental, control de la dieta por su estrecha relación con la caries que dependería de varios factores:

- Consistencia física de la dieta: cariogenicidad en relación a su adhesividad.

- Los alimentos son más cariogénicos entre las comidas, (sobretodo por las noches al descender la secreción salivar).

- La frecuencia de ingestión de alimentos es proporcional al incremento de caries.

Podemos establecer una escala de alimentos cariogénicos de más a menos según adhesividad, azucaramiento, hora de ingesta, etc..(Katz)

Evitar productos azucarados muy elaborados, facilitadores del acúmulo de placa que favorece la aparición de caries, abscesos, gingivitis, periodontitis, provocar una pérdida de inserción ósea del diente, la movilidad dental y la necesidad de extraer dientes fundamentales en la arcada dentaria con la consiguiente quiebra de calidad de vida.

Insistir en periódicas visitas al dentista, control de caries, obturaciones, aplicaciones tópicas de fluor, utilización de colutorios con fluor, tartrectomías, raspados, cureteados gingivales, uso de enjuagues con clorhexidina, control seriado radiográfico, etc. para un buen mantenimiento de la salud gingival y periodontal. 
Estos pacientes tienen marcada tendencia a maloclusiones de clase III, que se caracterizan por mesioclusión de los primeros molares inferiores, normalmente una oclusión invertida en el sector incisivo y dismorfosis esqueléticas asociadas.

El tratamiento va a depender de la etiología. En fase temprana podemos actuar de forma ortopédica sobre la estructura esquelética, según el caso, con mentonera, máscara facial, reguladores de función clase III; en fases más avanzadas con dentición mixta o definitiva, una actuación a nivel dentario además de la ortopédica en algunas fases del tratamiento, con aparatología removible, tipo placas con arco de progenie, placas con resortes de vestibulización, brackets, etc., en situaciones de dismorfias esqueléticas severas se recurre al tratamiento quirúrgico, cirugía ortognática, que permitiría la armonización de arcada superior e inferior.

La anodoncia parcial que presentan estos pacientes puede verse beneficiada del tratamiento ortodóncico que nos permitiría modificar la posición de algunos dientes en las arcadas dentarias, abriendo espacios o cerrando diastemas, con técnica multibrackets, utilizarlos como punto de apoyo para una rehabilitación protésica, utilizar implantes o minitornillos autorroscantes de titanio, como anclaje para movimientos retrusivos, intrusivos $(17,18)$.

En niños utilizamos placas de acrílico a las que añadimos dientes que permite una reconstitución estética, armónica, funcional, logrando un correcto desarrollo del hueso basal del maxilar hasta que se pueda realizar una prótesis implantosoportada.

En adultos el tratamiento protésico puede ser con prótesis dentomucosoportada, puentes adhesivos, implantes de titanio para rehabilitación unitaria o como pilar de puente en rehabilitaciones prostodóncicas fijas. El implante sufre una osteointegración y forma un todo con el hueso, como un diente anquilosado (19).

La identificación de familias portadoras de la mutación permite, a través del consejo genético, la prevención de la enfermedad.

\section{BIBLIOGRAFÍA}

1. García-Dorado J, De Unamuno P, FernándezLópez E, et al. Incontinentia pigmenti: XXY male with family history. Clin Genet 1990;38: 128-38.

2. Ormerod AD, White MI, McKay E, Johnston AW. Incontinentia pigmenti in a boy with Klinefelter's syndrome. J Med Genet 1987; 24: 439-41.

3. Lorda- Sanchez I, de Paula M, Bardaro T, Martín R, Villegas C, Ayuso C. Varón con incontinentia pigmenti asociada a síndrome de Klinefelter. An Esp Pediatr 2001; 55: 177-8.

4. The International Incontinentia Pigmenti (IP) Consortium.Genomic rearrangement in NEMO impairs NF-(kappa)B activation and is a cause of incontinentia pigmenti. Nature 2000; 405: 46672.

5. Aydingoz U, Midia M. Central nervous system involvement in incontinentia pigmenti: cranial MRI of two siblings. Neuroradiology 1998; 40: 364-6.

6. Urban J, Toruniowa B, Janniger CK, Czelej D, Schwartz RA. Incontinentia pigmenti (BlochSulzberger syndrome): multisystem disease observed in two generations. Cutis 1996; 58: 329-36.

7. Palencia R, Asensio D. Manifestaciones neurológicas en la incontinencia pigmenti (Síndrome de Bloch-Schulzberger). An Esp Pediatr 1994; 40: 146-8.

8. Landy SJ, Donnai D. Incontinentia pigmenti (Bloch-Schulzberger). J Med Genet 1993; 30: 53-9.

9. Rufo M, Sierra J.Facomatosis con discromías extensas: incontinencia pigmenti, nevus acrómico. Rev Neurol 1996; 24: 1060-7.

10. Pont V. Dermatosis lineales en la infancia. Piel 1997; 12: 89-97.

11. Francis JS, Sybert VP. Update on incontinentia 
pigmenti. Current Opin Dermatology 1995; 5560.

12. Kasmann-Kellner B, Jurin-Bunte B, Ruprech KW. Incontinentia pigmenti (Bloch-Schulzberger syndrome): case report and differential diagnosis to related dermato-ocular syndroms. Ophthalmologica 1999; 213: 63-9.

13. Rouse JS. Incontinentia pigmenti: overcoming cosmetic challenges. Compend Contin Educ Dent 1999; 20: 1034-6, 1040-1.

14. Carney RG. Incontinentia Pigmenti. A world statistical analysis. Arch Dermatol 1976; 112: 53542.

15. Yamashiro T, Nakagawa K, Takada K. Case Report: Orthodontic treatment of dental problems in incontinentia pigmenti. Angle Orthod 1998; 68: 281-4.

16. Tanboga I, Kargul B, Ergeneli S, Yalcin Aydin M, Atasu M. Clinical features of incontinentia pigmenti with emphasis on dermatoglyphic findings. J Clin Pediatr Dent 2001; 25: 161-5.
17. Maino BG, Pagin P, Mura P. Spider Screw $®$ : anclaje absoluto de carga inmediata. Rev Esp Ortod 2003; 33: 21-30.

18. Puigdollers A. Anclaje estable sobre implantes palatinos. Rev Esp Ortod 2003; 33: 121-31.

19. de Rábago JA, Sanz M. Manejo multidisciplinario de las agenesias dentarias. Periodoncia 2003; 13: 399-412.

\section{CORRESPONDENCIA}

Adolfo Pipa Vallejo.

Escuela de Odontología.

c/ Catedrático José Serrano, s/n. 33006 Oviedo.

Tfno.: 985103620

Fax: 984192261

Correo- electrónico: pipaadolfo@ uniovi.es. 